\title{
Seasat-A Satellite Scatterometer Instrument Evaluation
}

\author{
JAMES W. JOHNSON, LEON A. WILLIAMS, JR., EMEDIO M. BRACALENTE, FRED B. BECK, AND \\ WILLIAM L. GRANTHAM, MEMBER, IEEE
}

\begin{abstract}
The Seasat-A satellite scatterometer (SASS) was designed to measure ocean surface wind speed and direction in twenty-four (24) independent cells over a $1000-\mathrm{km}$ swath. It operated in the interrupted $\mathrm{CW}$ mode at a frequency of $14.6 \mathrm{GHz}$ with four (4) fan beam antennas and used Doppler filtering in the receiver for resolving the cells on the surface. The instrument began operating in space on July 6, 1978, and gathered normalized radar cross section $\left(\sigma^{\circ}\right)$ data for approximately $2290 \mathrm{~h}$. The purpose of this paper is to describe the in-orbit evaluation of the SASS hardware and its compatibility with the spacecraft. It has been determined that the scatterometer operated flawlessly throughout the mission, met all design requirements, and established a good data base for geophysical processing.
\end{abstract}

\section{INTRODUCTION}

$\mathrm{T}_{\mathrm{A}}^{\mathrm{H}}$ HE OBJECTIVE of the in-flight evaluation of the SeasatA scatterometer was to assure that the sensor hardware and related sensor data record (SDR) software were satisfactory for producing measurements of normalized radar cross section or scattering coefficient $\left(\sigma^{\circ}\right)$ and ocean surface wind vector. This was done primarily by using samples of data from the first 30 days of scatterometer operation. The credibility of the engineering unit software used to produce SDR's was first established and then the instrument performance was evaluated.

Radar scattering from the ocean surface has been a topic of interest to many investigators for several years [1]-[3], resulting in the proposition of using a microwave scatterometer technique for remotely sensing sea surface wind speed [4]. The empirical basis for the scatterometer measurement of ocean surface wind vector on Seasat-A was established by Jones et al. [5], and the scatterometer design requirements were described by Grantham et al. [6]. Consequently, the background material presented here is limited to the bare essentials of the characteristics and operation of the instrument. The SASS began operating on July 6, 1978, and an outline of the most significant scatterometer events over the mission is presented in Fig. 1. The bulk of this paper will deal with the functional validation and performance evaluation of the SASS. The methods used in the functional validation will be described and the results summarized. This will include a data quality discussion. The sources of error in a $\sigma^{\circ}$ measurement will be defined and those that are solely instrument related and require in-orbit evaluation will be identified. The performance evaluation discussion will address these error sources and will primarily consist of establishing the SASS receiver resolution capability and the best estimate of instrument stability. In addition, an RFI/EMI check will be presented. The engineering

Manuscript received October 31, 1979; revised May 10, 1980.

The authors are with NASA Langley Research Center, Hampton, VA 23665. unit level software validation is thoroughly discussed in [7] and will not be included here.

A companion paper [8] in this same issue gives a further description of the instrument's operation and signal processing details and describes the algorithms used to compute $\sigma^{\circ}$. Sample results of data processed to $\sigma^{\circ}$ are also given. It is intended that these two articles together will provide an understanding of the instrument operation, the data processing methods and the algorithms used for the computation of $\sigma^{\circ}$, and the overall performance of the instrument.

\section{INSTRUMENT DESCRIPTION}

The use of microwave scatterometers for the remote sensing of ocean surface wind speed and direction with the measurement of $\sigma^{\circ}$ over the ocean has been demonstrated in aircraft programs and in the Skylab S-193 experiment [9]. The following equation shows the functional relationship between $\sigma^{\circ}$ and the quantity sensed by the scatterometer $P_{R}$ :

$$
\sigma^{\circ}=\frac{P_{R}(4 \pi)^{3} R^{3}}{P_{T} \lambda^{2} L \phi L_{s} G_{0}{ }^{2}\left(\frac{G}{G_{0}}\right)^{2}}
$$

where

$$
\begin{array}{ll}
P_{R} & \text { received backscattered power } \\
P_{T} & \text { transmitted power } \\
R & \text { slant range } \\
\lambda & \text { free-space wavelength } \\
L & \text { Doppler cell footprint length in broad-beam plane } \\
L_{s} & \text { system and atmospheric losses } \\
\phi & \text { 3-dB beamwidth in narrow-beam plane } \\
G_{0} & \text { peak antenna gain } \\
G / G_{0} & \text { relative antenna gain at Doppler cell incidence angle. }
\end{array}
$$

Fig. 2 shows the scatterometer swath and nominal incidence angle distribution across the swath. The four fan beam antennas illuminated regions on the surface that were further subdivided by Doppler filtering into 12 cells each for wind vector measurements and three cells each for surface roughness measurements. The use of independent measurements from two antennas with orthogonal azimuthal viewing angles for each cell was required to infer both wind speed and wind direction [10] .

The scatterometer included the electronics package in Fig. 3 plus four antenna assemblies, each consisting of a horizontally and vertically polarized antenna pair as shown in Fig. 4 . The electronics package coupled to the antennas through eight separate waveguide sections. The block diagram of the scatter- 

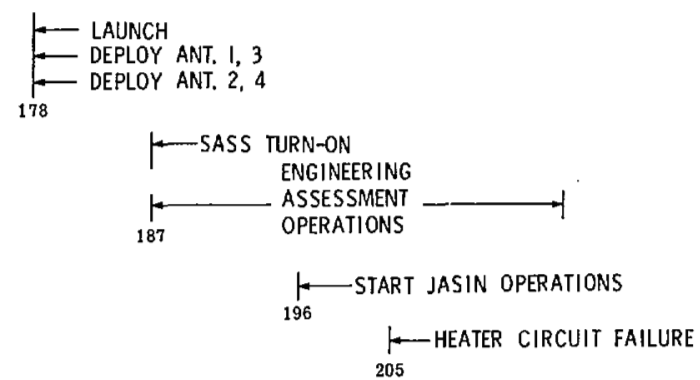
205

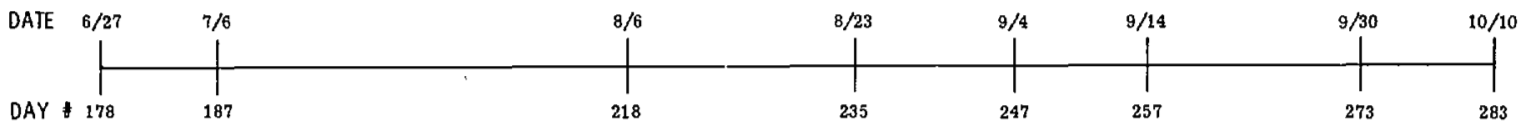

Fig. 1. Mission event flowchart.

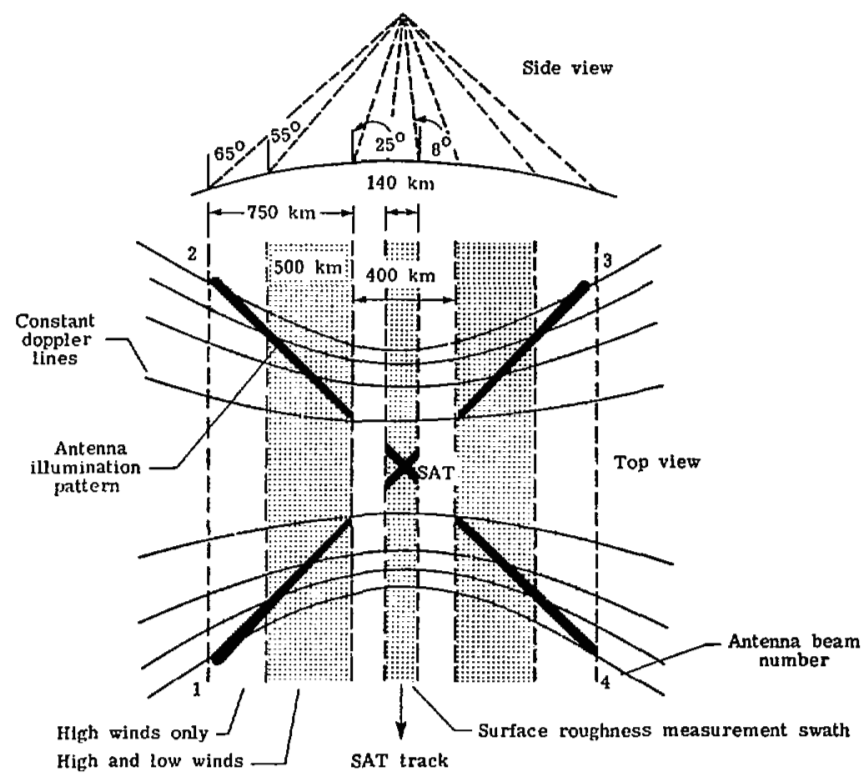

Fig. 2. Scatterometer measurement geometry.

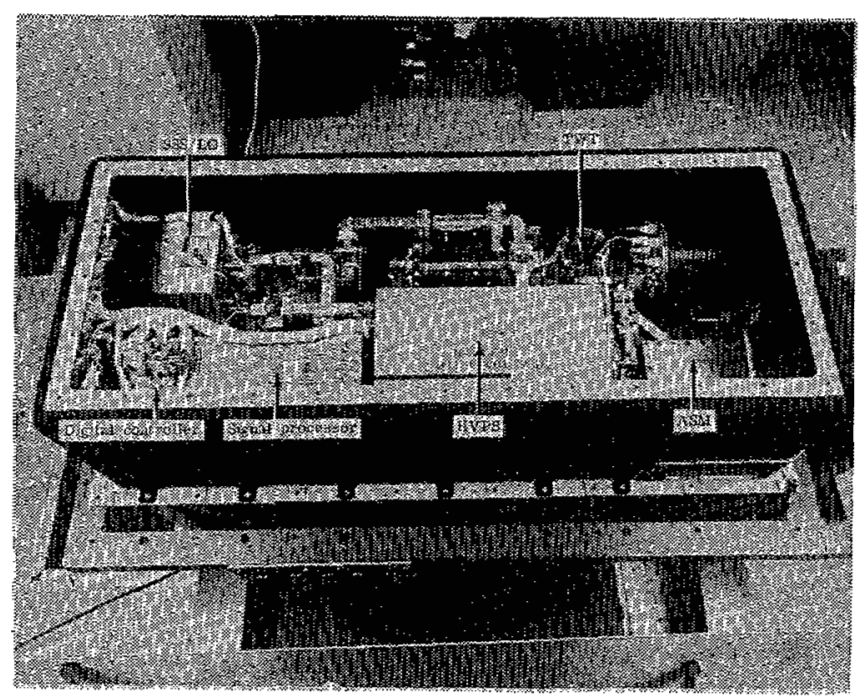

Fig. 3. SASS electronics package.

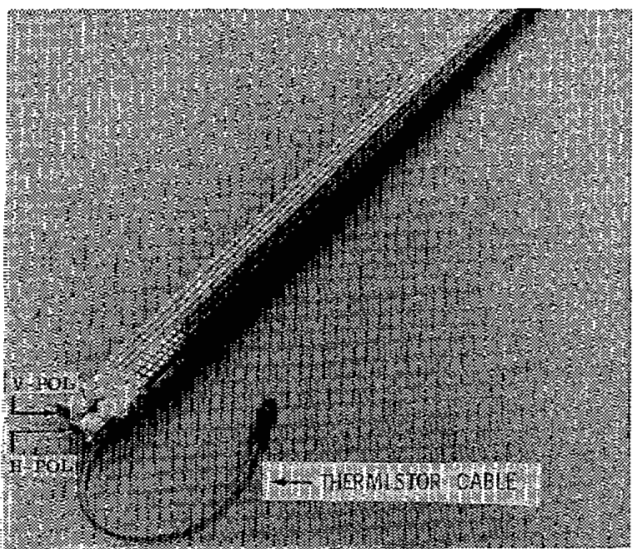

Fig. 4. SASS antenna assembly. 


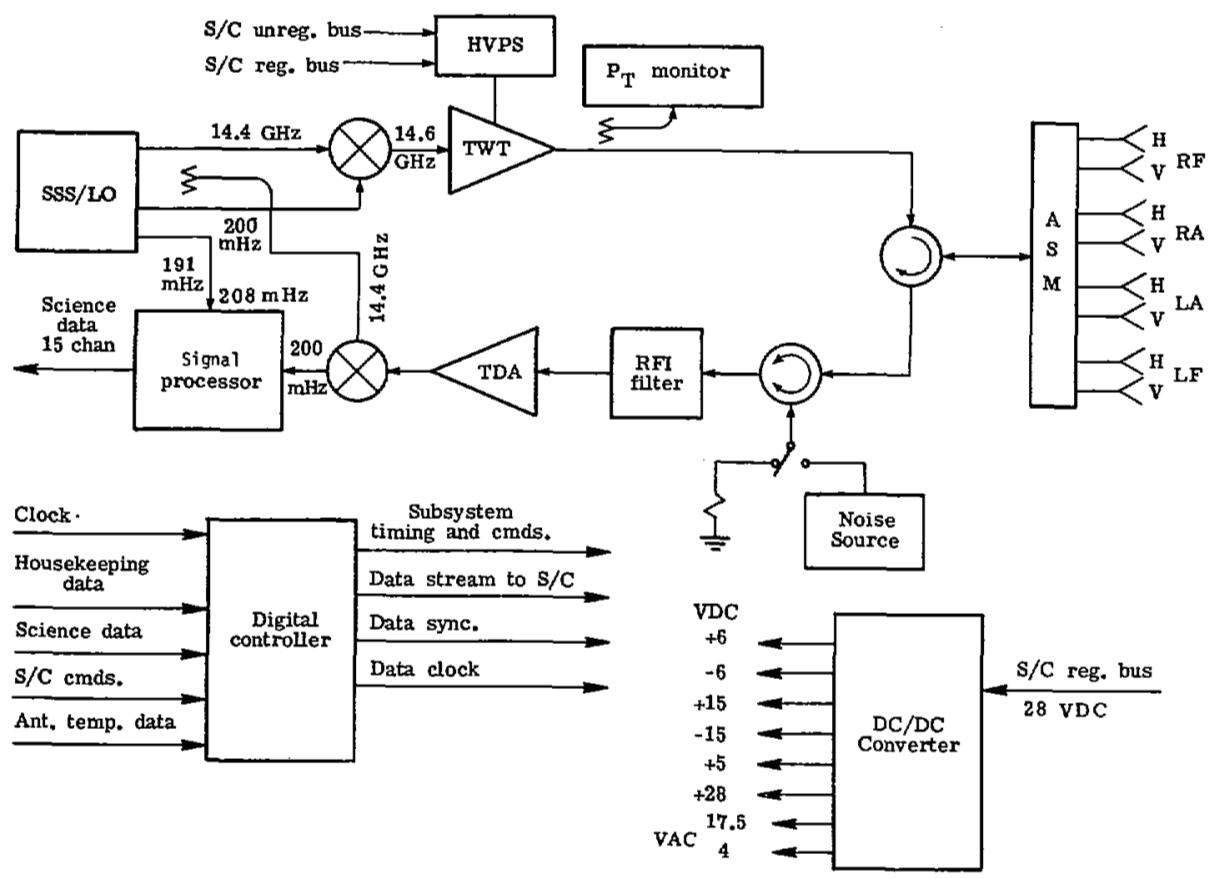

Fig. 5. SASS block diagram.

TABLE I

SASS MODE DESCRIPTION

\begin{tabular}{|c|c|}
\hline NODE & ANTDAXA SEQURECE \\
\hline 1 & $4 v, 2 v, 3 v, 2 v$ \\
\hline 2 & $4 \mathrm{H}, \mathrm{IH}, 3 \mathrm{H}, 2 \mathrm{ii}$ \\
\hline 3 & $4 \mathrm{~V}, 4 \mathrm{H}, 3 \mathrm{~V}, 3 \mathrm{H}$ \\
\hline 4 & $2 \mathrm{~V}, 2 \mathrm{H}, 2 \mathrm{~V}, 2 \mathrm{~S}$ \\
\hline 5 & $4 v, 4 v, 3 V, 3 V$ \\
\hline 6 & $2 \gamma, 2 V, 2 \nabla, 2 V$ \\
\hline 7 & $4 \mathrm{R}, 4 \mathrm{H}, 3 \mathrm{~K}, 3 \mathrm{H}$ \\
\hline 8 & $\mathrm{IH}, \mathrm{IH}, 2 \mathrm{H}, 2 \mathrm{~F}$ \\
\hline 9 & COMTIHUOUS CALIBRATE \\
\hline 10 & STAEDBY \\
\hline
\end{tabular}

TABLE II

NOMINAL INSTRUMENT CHARACTERISTICS

\begin{tabular}{|ll|}
\hline Operating Frequency & $14.59927 \mathrm{GHz}$ \\
Receiver Hoise Figure & $5.7 \mathrm{~dB}$ \\
Receiver Dymamic Range & $345 \mathrm{~dB}$ \\
Transqit Pulse Hidth & $4.8 \mathrm{msec}$ \\
Trarspitter Duty Factor & $17 \%$ \\
RF Output Pouer & 100 watts peak \\
DC Input Power & 136 watts \\
Height & $102 \mathrm{~kg}$ \\
Envelope Dimensions (Electronics) & $109 \times 48 \times 30 \mathrm{~cm}$ \\
\hline
\end{tabular}

ometer in Fig. 5 shows the major components and subsystems. The SSS/LO was a frequency synthesizer that provided the transmitter excitation at $14.6 \mathrm{GHz}$ and local oscillator signals to the receiver. The transmitted signal was interrupted $\mathrm{CW}$ at 17-percent duty factor with $100-\mathrm{W}$ peak power output from the TWT. The antenna switching matrix (ASM) selected the antenna for each set of backscatter measurements by switching in a periodic fashion according to the selected instrument operating mode (Table I). In all operating modes, 15 backscatter measurements were made every $1.89 \mathrm{~s}$ with an antenna switching cycle completed every $7.56 \mathrm{~s}$. This timing was designed to provide measurements which were located on a 50 $\mathrm{km}$ spacing in the along-track direction. The noise source provided a periodic receiver gain calibration every 250 s. A tunnel diode amplifier (TDA) was used for the first stage of amplification and set the receiver noise figure at less than 5.7 $\mathrm{dB}$ over the full receiver temperature range. Using range gating and Doppler filtering techniques in the signal processor, measurements were made of return signal power from the ocean surface plus background noise power $(S+N)$ and noise power only ( $N$ ONLY) for all 15 cells [8]. This produced 30 science data values every $1.89 \mathrm{~s}$ that, when corresponding pairs of $S+N$ and $N$ ONLY were processed, resulted in 15 measurements of $P_{R}$ which were proportional to $\sigma^{\circ}$. The digital controller used the spacecraft clock to generate the timing functions and commands required by the various subsystems, and it assembled the scatterometer data stream and interfaced it with the block formatted satellite data system. Table II is an overview of the instrument characteristics.

The SASS data stream consisted of 336 bits of housekeeping data (both bilevel and 10 bit words), 30 ten bit science words, and 184 sync and spare bits for a total of 820 bits. The 24 electronics package and 40 antenna assembly temperature monitors were subcommutated so that eight frames of data were required for a complete set of temperature measurements. The data stream was formatted serially and updated 
TABLE III

ERROR SOURCES IN THE MEASUREMENT OF $\sigma^{\circ}$

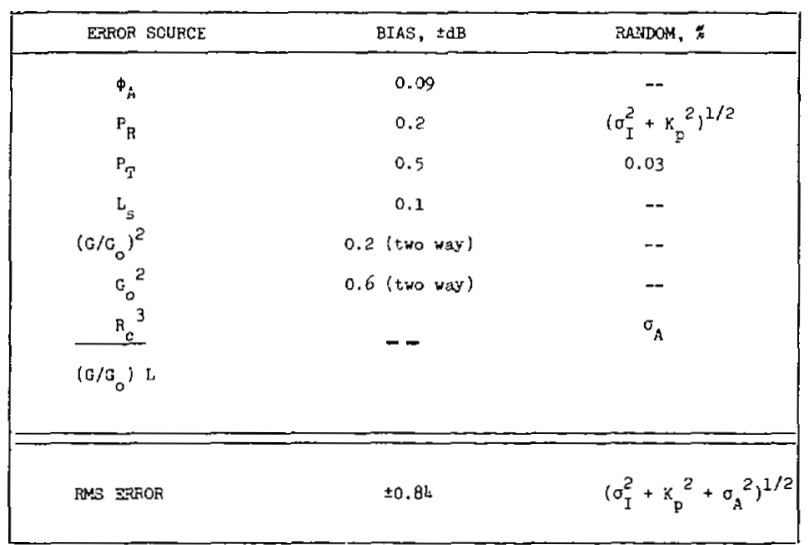

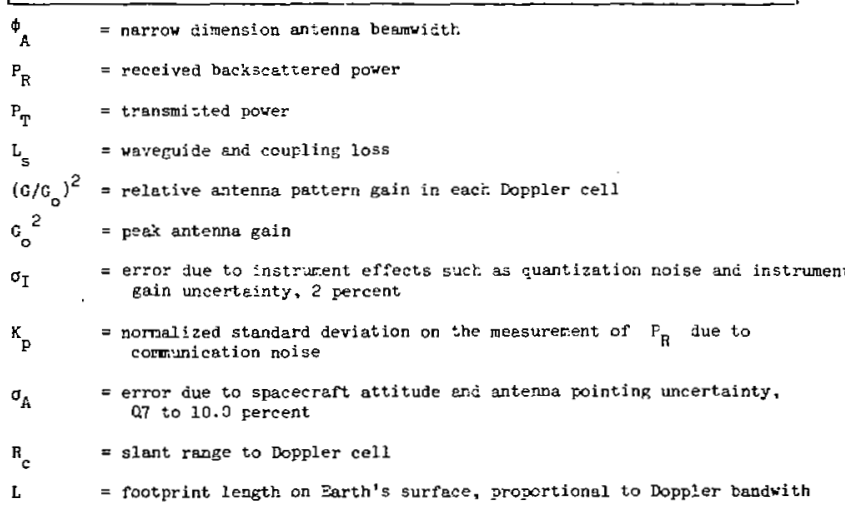

every 1.89 s. A complete list of all parameters in the scatterometer data stream along with the identification number for each and its location in a given frame is given in [7]

The spacecraft thermal design included heaters on the back of the scatterometer baseplate and radiation louvers attached to the edges of the baseplate, both aimed at maintaining the temperature between $0^{\circ} \mathrm{C}$ and $35^{\circ} \mathrm{C}$. The antennas were thermally isolated from the spacecraft.

\section{INSTRUMENT ACCURACY}

A variety of sources contributed to the potential error in the measurement of scattering coefficient. These are defined and listed in Table III as either bias errors or random errors. Each is discussed separately and the in-orbit evaluation required to address each is established in this section.

The quantities $\phi_{A}, G_{0}$, and $G / G_{0}$ were determined by antenna gain characteristic measurements prior to installation of the antennas on the spacecraft, and errors in the measurements were considered to be fixed bias errors. Errors in both $\boldsymbol{P}_{T}$ and $L_{s}$ were also fixed biases. The value of $L_{s}$ was measured in prelaunch component testing, and the \pm 0.5 - $\mathrm{dB}$ bias error on $P_{T}$ was derived from the accuracy limits on the prelaunch calibration of the TWT output power telemetry monitor. This monitor produced a voltage which was proportional to the integral of the peak transmit power and was updated in each data frame. Therefore, a value for $P_{T}$ averaged over a 1.89-s period was provided for each measurement of $\sigma^{\circ}$. The random error on the measurement of $P_{T}(0.03$ percent $)$ was considered negligible. The bias error in $P_{R}$ is due to uncertainty in the knowledge of the output power level of the calibration noise source used to determine and update receiver gain every $250 \mathrm{~s}$. Random errors in $P_{R}$ included those due to quantization noise and uncertainty in the measurement of receiver gain from the periodic calibrations $\left(\sigma_{I}\right)$ and communication noise $\left(K_{p}\right)$. The value of $\sigma_{I}$ was approximately 2 percent and $K_{p}$ was a function of the signal to noise ratio $S / N$ at the receiver.

Any uncertainty in the spacecraft attitude or in the antenna squint angle (i.e., narrow-beam dimension pointing) translated to errors in the footprint location parameters $R_{c}$ and $L$ and in the value of $G / G_{0}$. Errors in these quantities directly impact the accuracy of a $\sigma^{\circ}$ measurement according to (1). The attitude of the spacecraft and the squint angle varied slowly throughout each orbit. The attitude was continuously monitored, and a squint angle correction was routinely made based on antenna temperature, which was also continuously monitored. There were no applicable fixed bias error estimates for attitude and pointing effects; therefore, the total error $\sigma_{A}$ was considered random. The magnitude of $\sigma_{A}$ was different for each Doppler cell and each antenna beam, plus it was dependent on spacecraft latitude and longitude. Table III lists the maximum and minimum values that bound $\sigma_{A}$ for the mission. These were derived from an error in squint angle after temperature compensation of less than $0.1^{\circ}$ (Section IV) plus the results of an attitude uncertainty analysis that is fully discussed in [8].

As seen in Table III, the total rms bias error in the measurement of $\sigma^{\circ}$ was less than $\pm 0.84 \mathrm{~dB}$. Since this quantity was considered fixed, in-situ wind vector measurements used in satellite data/surface observation comparisons were used to correct a table which related $\sigma^{\circ}$ to wind speed, thereby removing bias effects on the wind vector measurement. One objective of the instrument performance evaluation was to confirm that these biases were indeed fixed. This was done using $\sigma^{\circ}$ data taken over the Amazon rain forrest to check both longterm ( $<53$ days) and short-term $(<150$ s) instrument stability (Section IV).

Since the SASS had 4 dual polarized antennas, there were 8 different antenna dependent biases, each within the $\pm 0.6-\mathrm{dB}$ value for $G_{0}{ }^{2}$. It was desirable to determine the relative bias between antennas since both a forward and aft measurement were required for the determination of wind speed and direction. These relative biases were determined using the Amazon rain forest data and are discussed in [8].

Corrections could not be made for random errors. Therefore, they were the most critical to the measurement accuracy with the error terms $K_{p}$ and $\sigma_{A}$ dominating. A more detailed discussion of the $K_{p}$ term as well as $\sigma_{A}$ is given in the companion paper [8]. Under low $S / N$ conditions, the value of $K_{p}$ approached $3 \mathrm{~dB}$, in the worst case, and dominated all other error sources. For high $S / N$ conditions, $K_{p}$ asymptotically approached its minimum value equal to $1 / \sqrt{B T}$, where $B$ is the Doppler cell bandwidth and $T$ is the measurement integration time. The minimum value of $K_{p}$ varied from 1 percent to less than 3 percent over all 15 measurement cells and was of the same order as the other random errors. The total rms random error due to all sources under high $S / N$ conditions was 
less than 11 percent $(0.47 \mathrm{~dB})$. Another objective of the instrument performance evaluation was to check the receiver resolution in orbit by determining the value of the instrument related contributions to the total random error. This was done by comparing the ratio of the standard deviation to the mean value of the output voltage in each Doppler channel, with constant input power, to the value of $1 / \sqrt{B T}$ (Section IV).

\section{HARDWARE EVALUATION}

\section{A. General}

The hardware evaluation was primarily based on data from the first 30 days of sensor operation. An engineering or functional evaluation was made using 88 percent of the scatterometer data gathered during this 30 -day period. In addition, the receiver resolution and instrument stability were determined in order to define the $\sigma^{\circ}$ measurement accuracy in terms of both instrument related random errors and the stability of the total bias error. An RFI/EMI study was also conducted.

\section{B. Data Quality}

Once SASS SDR's became available it was apparent that the bit error rate was high because of data handling at the ground receiving stations. An error analysis was performed using a limit test on three static SASS 10 bit words whose bit patterns and decimal equivalent values were fixed. Out of a data set of $1.45 \times 10^{6}$ samples, 273 errors were detected. To judge data quality for the scatterometer, this must be translated to the probability of an error in $\sigma^{\circ}$. Parameters that couple to the $\sigma^{\circ}$ computation must be factored into the error rate estimate with proper weighting [7]. For example, a single error in the value of transmit power translates to $15 \sigma^{\circ}$ errors, one in each measurement cell. After factoring in all parameters used in a $\sigma^{\circ}$ computation with each properly weighted, the probability of error is one in 16. As a result, tests designed to correct for bit errors were included in the $\sigma^{\circ}$ algorithm. For example, the limit test $P_{T}=96-101 \mathrm{~W}$ is performed on each value of transmit power; and, if the test is failed, the value from a latest available data table is substituted. These corrections reduced the probability of a $\sigma^{\circ}$ error to approximately $5 \times$ $10^{-4}$.

\section{Functional Validation}

Records at least 4 min long in each of the 10 operating modes were inspected for correct instrument status indication and for the cross correlation of related engineering parameters, such as TWT cathode voltage and transmit power. Data summaries that included all SASS data were also reviewed to verify execution of all commands and to monitor the mean value and standard deviation of all analog housekeeping parameters. The estimated mean value of each analog engineering parameter taken over this entire period is given in the "Key Parameter Matrix" in [7]. All electrical parameters were stable throughout the first 30-day period except for expected temperature effects, none of which affected system level performance. Both the transmit power and the receiver noise figure agreed with prelaunch test values and were stable. The instrument met all goals and specifications and duplicated its prelaunch behavior.

When the SASS was turned on, its baseplate temperature
TABLE IV

AMAZON DATA COMPARISON FOR LONG-TERM INSTRUMENT STABILITY

\begin{tabular}{|ccc|}
\hline ANT.H & Early Morning & Afternoon \\
\hline $1 \mathrm{~V}$ & $.10, \mathrm{~dB}$ & $.09, \mathrm{~dB}$ \\
$1 \mathrm{H}$ & .07 & .57 \\
$2 \mathrm{~V}$ & .33 & .13 \\
$2 \mathrm{H}$ & .16 & .35 \\
$3 \mathrm{~V}$ & .36 & -30 \\
$3 \mathrm{H}$ & .18 & ONLY 1 PASS \\
$4 \mathrm{~V}$ & .41 & .03 \\
$4 \mathrm{H}$ & .02 & ONLY 1 PASS \\
\hline
\end{tabular}

was approximately $3^{\circ} \mathrm{C}$, and, as all of the sensors began operating, the temperature increased to $22^{\circ} \mathrm{C}$ and stablized. After 18 days of operation, a satellite thermostat failure caused the median baseplate temperature to drop to $0^{\circ} \mathrm{C}$, which was a minimum for the mission. The lowest temperature reached on any subsystem was $-10.7^{\circ} \mathrm{C}$ on the ASM.

The antenna temperatures were generally lower than expected but never exceeded the $-90^{\circ} \mathrm{C}$ qualification test level Due to spacecraft shadowing, the inboard antennas ( 3 and 4) were cooler than the outboard antennas ( 1 and 2) by as much as $25^{\circ} \mathrm{C}$ to $30^{\circ} \mathrm{C}$. Since the antennas were thermally isolated from the satellite, the temperature varied over the length by as much as $15^{\circ} \mathrm{C}$ and the temperature at a given point varied by as much as $11^{\circ} \mathrm{C}$ over a complete orbit.

Corrections to the antenna squint angle due to thermal effects were made in the instrument data processing prior to computing the footprint location parameters in order to minimize the $\sigma_{A}$ random error term. Both the bulk temperature and the front-to-back temperature difference affected the narrow-beam squint angle. Corrections of $0.1^{\circ}$ or less in squint angle were required throughout the mission to correct for both temperature effects, with the front-to-back differential dominating.

\section{Receiver Resolution}

The scatterometer science data at the SDR level consisted of a set of output voltages proportional to received power. Each frame of data included 15 measurements with signal and noise present $(S+N)$ and 15 measurements with noise only present ( $N$ ONLY). It can be shown that for a fixed receiver input power, and for negligible quantization noise, the ratio of the standard deviation on the estimate of received power to the true value is approximately equal to the ratio of the standard deviation on the output voltage to the mean value of the voltage and is given by

$$
\frac{\sigma_{v}}{\mu_{v}}=\frac{1}{\sqrt{B T}}
$$

where $B$ and $T$ are the bandwidth and integration time, respectively [11]. As the record length or the number of data points averaged increases, this relationship approaches an equality. As discussed in Section III, for sufficiently high $S / N$ the scatterometer communication noise term $K_{p}$ approached a minimum value equal to $1 / \sqrt{B T}$. Consequently, (2) was used to evaluate the receiver performance in terms of its ability to 


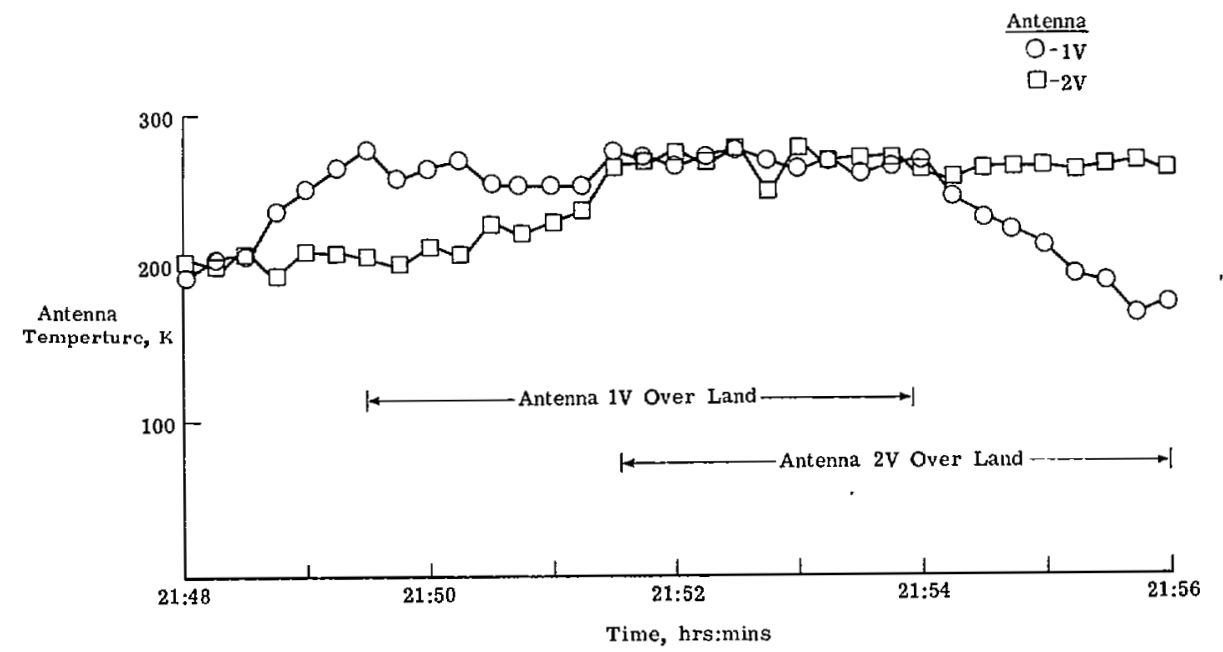

Fig. 6. Typical land/water radiometric antenna temperature profiles.

resolve changes in $P_{\gamma}$ under high $S / N$ conditions by estimating the noise added by instrument effects to the minimum value for $K_{p}$. Data was taken with the instrument in the STANDBY mode to approximate constant input conditions for averaging both $S+N$ and $N$ ONLY voltages over 20 data points each (approximately $38 \mathrm{~s}$ ). It was found that in all 15 Doppler channels the normalized standard deviation on the output voltage agreed with the computed value of $1 / \sqrt{B T}$. to within 0.55 percentage points. In all cases, the measured value was less than 3 percent, which indicated that the receiver was quite stable with a resolution under high $S / N$ conditions of better than $0.1 \mathrm{~dB}$.

These measurements reflected the $B T$ product contribution plus the quantization noise contribution, and confirmed that no other instabilities existed. The precision with which the receiver detector characteristics were defined prior to launch and the precision on the calibration noise voltage measurement were the limiting factors in the gain uncertainty contribution to $\sigma_{I}$. These effects were a function of received power and therefore random, but they could not be measured in flight. The prelaunch estimate of both quantization noise and gain uncertainties was 2 percent (Table III).

All contributions to the receiver resolution or the random error on $P_{R}$ under high $S / N$ have been considered and their magnitudes determined from flight data when possible. The rms value for the total random error on $P_{R}$ is less than 3.6 percent $(0.15 \mathrm{~dB})$ for high $S / N$.

\section{E. Instrument Stability}

Skylab data taken over the Brazilian rain forest demonstrated a spatial uniformity in $\sigma^{\circ}$ at $13.9 \mathrm{GHz}$ [12]. In addition, the rain forest is believed to be isotropic and polarization insensitive with respect to $\sigma^{\circ}$ since the foliage is extremely heavy and randomly oriented. With this in mind, SASS data for 11 orbits from revs 163-952 over the Amazon jungle was used to study both short-term and long-term instrument stability, including the stability of the fixed bias error terms. The Seasat $\sigma^{\circ}$ data supports the Skylab conclusion that the rain forest is homogeneous [8]. Significant differences in $\sigma^{\circ}$ properties were observed in data taken on early morning passes compared to late morning and afternoon passes; therefore, they must be considered separately in this analysis.
The method used to analyze the Amazon data involved averaging all $\sigma^{\circ}$ values on a given pass over the rain forest for each measurement cell and each antenna and polarization. The instrument was operating under relatively high $S / N$ conditions; therefore, the standard deviation on each set of $\sigma^{\circ}$ values normalized to the mean value should approach the minimum $K_{p}$, or instrument noise, when all other error contributors are negligible. For measurement cells near $43^{\circ}$ incidence angle, antenna pointing uncertainty effects were minimal and the normalized standard deviation on 20 point averages was $\leqslant 7$ percent, which is reasonably close to the computed value of $K_{p}$ for high $S / N$. This indicates that the instrument was stable over time periods in the 150-s neighborhood.

The mean values of $\sigma^{\circ}$ over as many as three orbits were also compared in order to get a worst case indication of longterm stability. Averages were computed for Doppler cells near $43^{\circ}$ incidence, and compared for all eight antenna paths (Table IV). The differences in the average value of $\sigma^{\circ}$ between different passes were due to both instrument effects and variability in target characteristics, but the available data given in Table IV indicate that the long-term ( $<53$ days) instrument stability is better than a few tenths of a dB. The variability in the instrument bias errors is within the probable variability of the target.

\section{F. RFI/EMI Effects}

In order to determine the RFI/EMI effects on the scatterometer, conventional radiometric antenna temperature $\left(T_{A}\right)$ measurements were made with and without the other Seasat sensors operating. The scatterometer was capable of measuring radiometric temperature to a resolution of approximately 12 to $30 \mathrm{~K}$. The value of $T_{A}$ was computed from each scatterometer noise measurement by the following:

$$
T_{A}=T_{E}-(L-1) T_{\mathrm{ASM}}-L T_{R}
$$

where

$T_{E} \quad$ measured effective input noise temperature

$T_{\text {ASM }} \quad$ ASM temperature

$T_{R}$ receiver noise temperature (TDA noise figure $=$ $5.5 \mathrm{~dB})$

$L \quad$ ASM path loss ratio. 


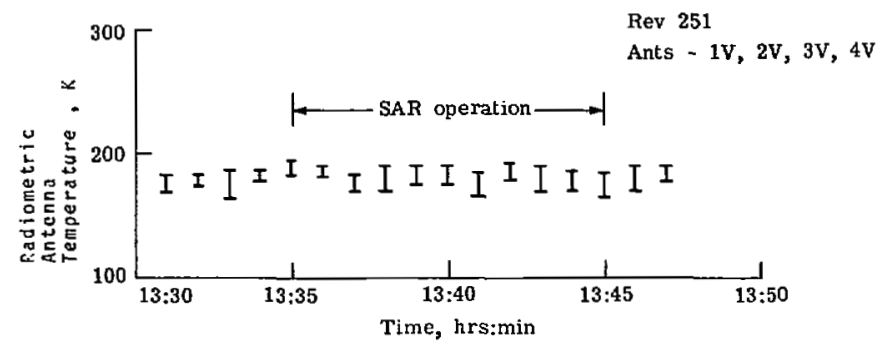

Fig. 7. Typical RFI/EMI test results.

$T_{A}$ was routinely computed for the $N$ ONLY measurement in each channel. Since the signal was broad-band noise, there was no spatial discrimination by filtering, and the target was defined by the entire antenna footprint. Therefore, $T_{A}$ in each receiver channel varied as the bandwidth and integration time only and was a function of the surface radiation properties over a wide range of incidence angles. The values for $T_{A}$ reported here were averaged over all 15 cells. The objective was to determine the RFI effects of the other sensors on the average noise level into the scatterometer, and the approach was to establish baseline values for $T_{A}$ with only the SASS on the then draw comparisons with all sensors on.

On rev 141 as the satellite crossed over the northern end of South America, the instrument was operating in STANDBY using antennas $3 \mathrm{~V}$ and $4 \mathrm{~V}$. No other sensors were on. Averaging over 20 values, all taken over water with only the receiver on, $T_{A}$ for the $3 \mathrm{~V}$ path was $184 \mathrm{~K}$ and for the $4 \mathrm{~V}$ path was $174 \mathrm{~K}$. The difference was due to $0.1 \mathrm{~dB}$ of ASM path loss difference. These values were typical of all antennas and established a baseline where the entire footprint was viewing water and neither the SASS transmitter nor any of the other sensors was operating.

The instrument began transmitting in mode 4 as it approached land. Fig. 6 shows $T_{A}$ profiles for antennas $1 \mathrm{~V}$ and $2 \mathrm{~V}$ with transitions from water to land. The forward looking antenna ( $1 \mathrm{~V}$ ) was the first to level off at approximately $270 \mathrm{~K}$ with a land filled footprint followed by the aft looking antenna (2V). After approximately $5 \mathrm{~min}$, the forward footprint was again over water and $T_{A}$ was returning to the neighborhood of 170 to $180 \mathrm{~K}$. This established baseline data over land and water with the SASS transmitter on, and indicated that the transmitter itself caused neither EMI nor RF coupling effects.

Fig. 7 shows a typical set of RFI data where all sensors were on, including the synthetic aperture radar (SAR), for 10 $\min$. In this case all antennas were viewing only water and the data spread was due primarily to ASM path loss differences. All values were in the 170 to $180 \mathrm{~K}$ region, indicating no apparent RFI or EMI effects with all sensors operating. Data in other operating modes using the horizontally polarized antennas were typically less than $270 \mathrm{~K}$ over land and less than 180 $\mathrm{K}$ over water, again demonstrating no measurable effects from other sensors.

\section{SUMMARY}

It has been determined that the SASS hardware and software performed well during the Seasat mission and that SASS sensor data records are acceptable for geophysical processing.

The SASS hardware performed flawlessly for $2290 \mathrm{~h}$, meeting all of its design goals and specifications. A satellite heater circuit failure caused the electronics package to operate close to its design limit $\left(0^{\circ} \mathrm{C}\right)$ for most of the mission, and the antenna temperatures were lower than expected. However, instrument performance is not believed to have been affected. All engineering housekeeping parameters were stable at their prelaunch values. The transmitter was stable and operated at full output power, and the receiver noise figure agreed with prelaunch values. Receiver resolution tests determined that no measurable contributors to the instrument related random error existed, other than quantization noise and receiver gain uncertainty. The total random error under high $S / N$ conditions, including attitude uncertainty, was estimated to be less than $0.47 \mathrm{~dB}$, and under worst case $S / N$ conditions approached $3 \mathrm{~dB}$. Data taken over the Amazon rain forest indicated that the fixed bias errors, which were removable, were stable to a few tenths of a $\mathrm{dB}$ or less.

\section{ACKNOWLEDGMENT}

The authors wish to acknowledge the support of a host of other people to the scatterometer operation during integration and system testing, in the development of software and operations planning prior to launch, and in operations and data processing during and after the mission. People from the Jet Propulsion Lab (JPL), Goddard Space Flight Center, and the Lockheed Missiles and Space Company (LMSC) as well as the scatterometer contractors contributed to the effort. Particular thanks are due Art Heath and Attie Salamon of the General Electric Company for their assistance throughout, especially during satellite system tests. Special thanks also to John Schlue and Carl Kloss of JPL and Vince Moughan of LMSC.

\section{REFERENCES}

[1] N. W. Guinard and J. C. Daley, "An experimental study of sea clutter model," Proc. IEEE, vol. 58, pp. 543-550, Apr. 1970.

[2] G. R. Valenzuela, M. B. Laing, and J. C. Daley, “Ocean spectra for the high frequency waves as determined from airborne radar measurements," J. Mar. Res., vol. 29, no. 2, pp. 69-84, May 1971.

[3] G. A. Bradley, "Remote sensing of ocean winds using a radar scatterometer," Ph.D. dissertation, University of Kansas Center for Research, Inc., Sept. 1971.

[4] R. K. Moore and W. J. Pierson, Jr., "Worldwide oceanic wind and wave predictions using a satellite radar radiometer, "J. Hydronaut., vol. 5, no. 2, pp. 52-60, Apr. 1971.

[5] W. Linwood Jones, L. C. Schroeder, and J. L. Mitchell, "Aircraft measurements of the microwave scattering signature of the ocean, "IEEE Trans. Antennas Propagat./IEEE J. Oceanic Eng. (Special Issue on Radio Oceanography), pp. 52-61, Jan. 1977.

[6] William L. Granthan, Emedio M. Bracalente, W. Linwood Jones, and James W. Johnson, "The Seasat-A satellite scatterometer," IEEE J. Oceanic Eng., vol. OE-2, pp. 200-206, Apr. 1977.

[7] James W. Johnson, Wendell H. Lee, and Leon A. Williams, Jr., "Seasat-A satellite scatterometer mission summary and engineering assessment report," NASA TM 30122, May 1979.

[8] E. M. Bracalente, D. H. Boggs, W. L. Grantham, and J. L. Sweet, "The SASS scattering coefficient $\left(\sigma^{\circ}\right)$ algorithm," this issue, pp. 145-154.

[9] R. K. Moore and A. K. Fung, "Radar determination of winds at sea," Proc. IEEE, vol. 67, pp. 1504-1521, Nov. 1979.

[10] W. L. Grantham, E. M. Bracalente, W. L. Jones, J. H. Schrader, and J. L. Mitchell, "An operational satellite scatterometer for wind vector measurements over the ocean," NASA TM X-72672, Mar. 1975.

[11] A. F. Harvey, Microwave Engineering. London and New York: Academic, 1963, p. 777.

[12] A. Sobti and E. C. Davison, "Microwave scattering measurements over Brazil at $13.9 \mathrm{GHz}$," University of Kansas Remote Sensing Lab Tech. Rep. 243-11, Sept. 1975. 